\title{
On Structure Preserving Quantisations
}

\author{
T. S. SHANKARA \\ Mathematical Sciences Division, National Aerorautical Laboratory, \\ Bangalore-17 (India) \\ and \\ M. D. SRINIVAS \\ Physics Department, Central College, Bangalore Unstersity, \\ Bangalore-I (India)
}

Received: 14 April 1971

\section{Abstract}

Maps of functions on classical phase space to quantum operators do not preserve the algebraic structure. After locating the algebraic reasons for it, the problem of quantisation is redefined and the Moyal bracket is discussed for its structure preservation. This quantisation entails the inclusion of Schwartz distributions to the space of classical functions.

\section{Introduction}

Consider the phase space $Z=(q, p)$ of one-dimensional non-relativistic motion. Let $C(z)$ be the set of classical physical observables which are infinitely differentiable functions on (z). Now $C$ is a Lie algebra under the Poisson bracket

$$
(f, g)_{\text {P.s. }}=\frac{\partial f}{\partial q} \frac{\partial g}{\partial p}-\frac{\partial f}{\partial p} \frac{\partial g}{\partial q} .
$$

By a quantisation of $C$ is meant the determination of a linear map $E: C \rightarrow C$ of self-adjoint operators on the Hilbert space of state vectors such that

$$
E(f, g)_{\text {r.s. }}=[E f, E g]
$$

where the right-hand side is the conmutator of $E f$ and $E g$. This rather old problem is sufficiently battered about by now with regard to its algebraic structure preservation; one concludes (Arens \& Babbitt, 1965) that it is not possible to find an $E$ such that

$$
E(f \cdot g)=E f E g
$$

Copyright 1971 Plenum Publishing Company Limited. No part of this publication may be reproduced, stored in a retrieval system, or transmitted, in any form or by any means, electronic, mechanical, photocopying, microfilming, recording or otherwise, without written permission of Plenum Publishing Company Limited. 
where $f \cdot g(z)=f(z) g(z)$ is the pointwise product of $f$ and $g \in C$. Now in retrospect we feel that trying to solve (1.3) for $E$ must indeed be infructuous for the following reason:

In apposition to classical mechanics, physical observables in quantum theory, namely elements of $\bar{C}$, are stochastic variables with probability distribution functions which have positice dispersion in general. Suppose we want the joint probability distribution of two stochastic variables to be given uniquely from their marginal probability distributions alone. This is pussible if and only if the operators corresponding to these stochastic variables commute (von Neumann, 1955). Hence in a semi-classical description where we try to embed these stochastic variables in a function space of probability distributions in phase space, it is necessary that these functions constitute a non-commutative algebra. But the point product $f . g$ in (1.3) is commutative and even the functions are causal functions. Hence, it looks reosonable to postulate instead an equation such as

$$
E(f \times g)=E F E G
$$

where $f \times g$ is a suitable non-commutative product of classical "objects", whose nature we shall determine in the last section, and $F$ and $G$ are in some way related to $f$ and $g$ respectively.

In fact it is important to note that non-commutativity is not sufficient; in order that $E$ be a homomorphism the algebra should also be associative, because the algebra on $C$ is associative. For instance, consider the Poisson bracket. It has been proved (Wollenberg, 1969) that it cannot be represented by a commutator over an associative algebra on $C(z)$. it is precisely for this reason that there does not exist an $E$ satisfying the equation (1.2).

Hence we propose, in this paper, to discuss quantisation of such noncommutative associative algebras. We shall first carry out this programme in a general set up and then deal with the Moyal algebra as a particular case, which, in our opinion, is the only relevant one.

\section{The General Case}

On $C$ define the product

$$
f \times g=\int K\left(z_{1}, z_{2}, z\right) f\left(z_{1}\right) g\left(z_{2}\right) d z_{1} d z_{2}
$$

through a kernel $K \in C$. (Here and hereafter all the integrals are from $-\infty$ to $+\infty$.) For $C$ to represent a dynamical system we must require (2.1) to fulfil the concitions that

(a) $C$ is closed under $x$;

(b) $x$ is not commutative in general;

(c) $x$ is associative. 
Then there exists the natural Lie bracket $\{f, g\}=f \times g-g \times f$ on $C$ and the mapping $g \rightarrow\{f, g\}$ is a derivation over $C$.

These requirements on the product naturally throw up sufficient conditions on $K$ in the form of functional equations (Sriram \& Shankara, 1969). Hence for a suitable class of kernels $K \in C$ for which the corresponding functional equations are solvable, we have in $C$ a representation of dynamics.

Now we shall determine an algebra homomorphism $E: C \rightarrow C$; in other words, we shall find a mapping $E$ such that

$$
E\{f, g\}=[E F, E G] .
$$

Allowing $E$ to operate on (2.1) we have

$$
\begin{aligned}
E(f \times g) & =E \int K\left(z_{1}, z_{2}, z\right) f\left(z_{1}\right) g\left(z_{2}\right) d z_{1} d z_{2} \\
& =\int E K\left(z_{1}, z_{2}, z\right) f\left(z_{1}\right) g\left(z_{2}\right) d z_{1} d z_{2} .
\end{aligned}
$$

Assume that there exists a factorisation

$$
E K\left(z_{1}, z_{2}, z\right)=E K_{\alpha}\left(z_{1}, z_{2}, z\right) E K_{\beta}\left(z_{1}, z_{2}, z\right)
$$

where $K_{\alpha}, K_{\beta} \in C$. Then the above equation gives

$$
E(f \times g)=\int E K_{\alpha} f\left(z_{1}\right) d z_{1} \int E K_{\beta} g\left(z_{2}\right) d z_{z} .
$$

Now define

$$
\begin{aligned}
& F=\int K_{\alpha}\left(z_{1}, z_{2}, z\right) f\left(z_{1}\right) d z_{1} \\
& G=\int K_{\beta}\left(z_{1}, z_{2}, z\right) g\left(z_{2}\right) d z_{2}
\end{aligned}
$$

as $K$-integral transforms of $f$ and $g$ respectively. Then (2.4) assumes the form

$$
E(f \times g)=E F E G
$$

Since $K, K_{\alpha}, K_{\beta}$ all belong to $C$ the functions $f \times g, F, G$, also belong to $C$. Hence $E$ is a homomorphism of $C \rightarrow C$. Of course, such an $E$ autcomatically ensures also the Lie algetura homomorphism:

$$
E\{f, g\}=E(f \times g-g \times f)=[E F, E G] .
$$

However, the assurned factorisation (2.3) is not unique; indeed any $E K_{\alpha}$ which has an inverse determines $E K_{\beta}$ and conversely. Hence the mapping $f \rightarrow F$ is one-to-many. 


\section{Homomorphic Quantisation of the Moyal Algebrat}

Now for an exampie, we shall deal explicitly with the quantisation of the Moyal algebra (Jordan \& Sudarshan, 196́l). The Moyal bracket is

$$
\{f, g\}=f(z) \sin \left(\frac{\partial}{\partial q} \frac{\vec{\partial}}{\partial p}-\frac{\grave{\partial}}{\partial p} \frac{\vec{\partial}}{\partial q}\right) g(z)
$$

which can further be thrown into a commutator form as

$$
\{f, g\}=f \times g-g \times f
$$

where

$f \times g=\frac{!}{i} \int \exp \left\{i\left[\left(q-q_{1}\right)\left(p-p_{2}\right)-\left(q-q_{2}\right)\left(p-p_{1}\right)\right\}\right\} f\left(z_{1}\right) g\left(z_{2}\right) d z_{1} d z_{2}$.

This is an associative, non-commutative, non-local product.

Now many of the operator assignments are representable as

$$
E f=\int \exp (i \eta \hat{q}+i \tau \hat{p}) \lambda(\eta, \tau) \exp (-i \eta q-i \tau p) f(z) d \eta d \tau d z
$$

where $\hat{q}$ and $\hat{p}$ are position and momentum operators respectively and $\lambda$ is a differentiable function satisfying some boundary conditions (Misra \& Shankara, 1968). Integrating the right-hand side we get

$$
\begin{aligned}
E f & =\int f(z) \cdot \mathscr{F} \lambda(\xi-z) d z \\
& =(f * \mathscr{F} \lambda)(z)
\end{aligned}
$$

where $\mathscr{F} \lambda$ is the Fourier transform of $\lambda$ and $*$ is the convolution. When $\lambda$ is a polynomial $\mathscr{F} \lambda$ is a distribution. For example Weyl's rule corresponds to the case $\lambda=1$, so that

$$
E_{w} f=(f * \hat{o})(z)=f(z)
$$

We shall restrict to this rule in our further discussion.

Now the operator corresponding to the product function (3.2) is hence given by

$$
\begin{aligned}
E_{w}(f \times g)= & \frac{1}{i} \int_{\times f\left(z_{1}\right) g\left(z_{2}\right) d z_{1} d z_{2}} \exp \left\{i\left[\left(\hat{q}-q_{1}\right)\left(\hat{p}-p_{2}\right)-\left(\hat{q}-q_{2}\right)\left(\hat{p}-p_{1}\right)\right]\right\} \times \\
= & \frac{1}{i} \int_{\times f\left(z_{1}\right) g\left(z_{2}\right) d z_{1} d z_{2} .} \exp \left\{i\left[\left(p_{1}-p_{2}\right) \hat{q}-\left(q_{1}-q_{2}\right) \hat{p}+\left(q_{1} p_{2}-q_{2} p_{1}\right)\right]\right\} \times
\end{aligned}
$$

$f$ After this paper was submitted, we received a Texas University preprint by Simoni. Sudarshan and Zaccaria in which the quantisation of non-commutative associative algebras has been solved. They show that apart from trivial transformations, the complex Moyal bracket (with $h$ complex) is the only solution: the classical Poisson bracket is the limit $\boldsymbol{h} \rightarrow \mathbf{0}$ of the Moyal bracket. 
The exponential operator in the integrand of this integral may now be factorised using Baker-Hausdorf formula. Thus

$$
\begin{aligned}
E_{w}(f \times g)= & \frac{1}{i} \int \exp \left[i\left(p_{1} \hat{q}-q_{1} \hat{p}+\frac{1}{2} q_{1} p_{2}\right)\right] f\left(z_{1}\right) d z_{1} \times \\
& \times \int \exp \left[-i\left(p_{2} \hat{q}-q_{2} \hat{p}-\frac{1}{2} q_{2} p_{1}\right)\right] g\left(z_{2}\right) d z_{2} \\
= & \frac{1}{i} E_{W} F E_{w} G
\end{aligned}
$$

where

$$
\begin{aligned}
& F=\int \exp \left[i\left(p_{1} q-q_{1} p+\frac{1}{2} q_{1} p_{2}\right)\right] f\left(z_{1}\right) d z_{1} \\
& \boldsymbol{G}=\int \exp \left[-i\left(p_{2} q-q_{2} p-\frac{1}{2} q_{2} p_{1}\right)\right] g\left(z_{2}\right) d z_{2} .
\end{aligned}
$$

Thus the assumption (2.3) of our hypothesis is satisfied. But the factorisation employed above is not unique; an obviously different splitting is obtained on interchanging the numbers $q_{1} p_{2}$ and $q_{2} p_{1}$ in the two exponentials in (3.4) and (3.5).

\section{Some Features of This Quartisation}

Consider the product (3.2). If $f$ and $g$ are both functions of either only $q$ or only $p$, it is easy to demonstrate that $f \times g$ is commutative. It is noncommutative only when there is a mix-up of $q$ and $q$ in the product. This is already reminiscent of the quantum situation. Hence, in this quantisation, it would be interesting to seek the classical image of the polynomial operators of quantum theory in particular. For this purpose it would be sufficient to consider the case of the fundamental commutator with $E F=\hat{q}$ and $\boldsymbol{E} \boldsymbol{G}=\hat{\boldsymbol{p}}$ which are the traditional position and momentum operators obtained by Weyls's rule. Thus it is required to determine $f$ and $g$ corresponding to $F$ and $G$. From (3.4) and (3.5) we have

$$
\begin{aligned}
& q=\int \exp \left[i\left(p_{1} q-q_{1} p+\frac{1}{2} q_{1} p_{2}\right) f\left(z_{1}\right) d z_{1}\right. \\
& p=\int \exp \left[-i\left(p_{2} q-q_{2} p-\frac{1}{2} q_{2} p_{1}\right)\right] g\left(z_{2}\right) d z_{2}
\end{aligned}
$$

Soiving these equations for $f$ and $g$ we get

$$
\begin{aligned}
f\left(z_{1}\right) & =\exp \left(-\frac{i}{2} q_{1} p_{2}\right) \int q \exp \left[-i\left(p_{1} q-q_{1} p\right)\right] d q d p \\
& =-i \exp \left(-\frac{i}{2} q_{1} p_{2}\right) \delta\left(q_{1}\right) \delta^{\prime}\left(p_{1}\right)
\end{aligned}
$$




$$
\begin{aligned}
g\left(z_{2}\right) & =\exp \left(-\frac{i}{2} q_{2} p_{1}\right) \int p \exp \left[i\left(p_{2} q-q_{2} p\right)\right] d q d p \\
& =-i \exp \left(-\frac{i}{2} q_{2} p_{1}\right) \delta^{\prime}\left(q_{2}\right) \delta\left(p_{2}\right) .
\end{aligned}
$$

Now these are Schwartz distributions astir $;$ or $\mathrm{kz}$ mel functions belonging to $C$ and not just ordinary functions. Th., ire tari shown that if quantisation is required to be homomorph:i, it will be necessary to represent classical observables by Schwariz distributions when the corresponding quantum observables are polynomiais if $z$. $t$. is is a result which completely corroborates the situation in Sudarsnan's 'Optical Equivalence Theorem (Sudarshan, 1963). But we shall ela':orate on this point elsewhere. As in Sudashan's theorem, at first jight, the presence of these distributions may appear to give rise to dificulties when their products are involved-a situation elso imitating the difficulties in Hermann's quantisation (Hermann, i965); namely, when he identifies the classical phase space with a susei of the quantum phase space by his quantisation (fractional), powers of the $\delta$-function appear which do not fit in with the standard Hilbert space framework. But here, towever, the definition of the product of distributions is valid since their domains are disjoint and the definition always includes the kernels which act as test functions. Thus the results of this section are in precise agreement with our speculations in the second paragraph of the introduction.

\section{Conclusion}

The multiplication of the base algebra over which the Poisson bracket is defined is non-associative, commutative and local; indeed, it cannol be expressed as a commutator over an associative algebra. The quantum mechanical Lie algebra on the other hand, is exactly its antithesis: namely, its Lie bracket is a commutator over an associative, non-commutative and non-local algebra. It is this situation which forbids any rule of quartisation from preserving the algebraic structure. Hence, if one insists on structure preservation, it is necessary that the base algebra of classical observables is also associative, non-commutative and non-local. In this paper such algebras have been quantised in general and the Moyal algebra is considered as a particular example. The procedure demonstrates that the elements of the algebra be Schwartz distributions acting on kernels whict .e ordinary functions, but are not ordinary functions themselves. Thus, an homomorphic quantisation enforces a prolongation of the ring of observables on classical phase space to include Schwartz distributions. Hence it would be interesting to derive the actual expressions of these classical distributions corresponding to various density matrices thet oceus in quantum theory and study them in the light of the Optical Equivalence Shescm. 


\section{Acknowledgements}

We thank Prof. E. C. G. Sudarshan for his kind comments. We are indebted to Dr. S. R. Valluri, Director, National Aeronautical Laboratory and Dr. K. N. Kunchela, Head of the Physics Department, Bangalore University, for their kind interest. We also thank Sri M. S. Sriram for interesting discussions.

\section{References}

Arens, R. and Babbitt, D. (1965). Journal of Mathematical Physics, 6, 1071. Hermann, R. (1965). Journal of Mathematical Physics, 6, 1768. Jordan, T. F. and Sudarshan, E. C. G. (1961). Review's of Modern Physics, 33, 515. Misra, S. P. and Shankara, T. S. (1968). Journal of Mathematical Physics, 9, 299. von Neumann (1955). Mathematical Foundations of Quantum Mechanics. Princeton University Press.

Sriram, M. S. and Shankara, T. S. (1969). Lettere ai Nuoto Cimento, 2, 567.

Sudarshan, E. C. G. (1963), Physical Reciew Letters, 10, 277.

Wollenberg. L. S. (1969). Journal of the London Mathematical Society, 44A, 592. 